IX.

Ein Neurom der ERB'schen Plexuswurzeln.

Operirt ron

Prof. Dr. E. Rose

in Berlin.

Recht auffallend ist das Bild der Erb'schen Wurzellähmung für den Chirurgen, wenn er auch noch soriel Lähmungen am Arm beobachtet hat, mag er es mit Stichwunden am Hals, mit Säbelhieben am Arm, mit Zerreissungen des Rückenmarks und der Armnerven bei Knochenbrichen, bei Oberarmverrenkungen, bei Kammradwunden am Oberarm, mit Neuromen am Arm und dergleichen mehr zu thun gehabt haben. In der Regel handelt es sich hierbei um unvollständige, von den Fingern nach oben auslaufende Lähmungsgebiete. Entsprechend der bekannten Versorgung der Finger durch die verschiedenen Armnervenstämme zusammen, leidet dann, zumal bei der in solchen Fällen mehr oder weniger unvollständigen Betheiligung der einzelnen Stämme, meist nur der Gebrauch einzelner Finger; oft sogar fehlen nur gewisse Functionen an den Fingern, während sich weiter hinauf am Arm Störungen noch weniger oder auch wohl gar nicht nachweisen lassen. Anders hier! Der Gegensatz zwischen der vollständigen Unbrauchbarkeit des Oberarms und der vollen Gebrauchsfähigkeit der Hand bei Erhaltung der Sensibilität macht die Erb'sche Wurzellähmung so uberaus überraschend, dass es vielleicht gerechtfertigt ist, eine operative Bestätigung für die anatomische Erklärung dieser so eigenthümlichen Lähmung mitzutbeilen, wenn auch der Fall in unserer Heilanstalt ihren eigenthümlichen Verbältnissen entsprechend in seiner längeren Beobachtung auf Hindernisse gestossen ist.

Bekanntlich hat Erb 1874 zuerst diese eigenthümlich begrenzte Lähmung der Schultermusculatur mit Erhaltung der Sensibilität in die oberen Wurzeln des Armgeflechts rom 5. und 6. Halsnerven und ibren gemeinsamen Stamm localisirt. Er fand dem entsprechend beim Gesunden am Halse einen Punkt, nden Erb'schen Punkt"1) (im Tri-

1) Nach Dr. ten Cate ist es der Kreuzungspunkt des Schlüsselbeins mit der 
gonum cervicale posticum $2-3 \mathrm{Cm}$. oberhalb der Clavicula aussen rom hinteren Rande des Kopfnickers in der Höhe des vorderen Tuberkels vom Querfortsatz des 6. Halswirbels), dessen elektrische Erregung die gleichzeitige Contraction des Deltoides, Biceps, Brachialis internus und Supinator longus, also die Fülurung der Hand zum Kopf hervorbringt.

Freilich sind solche isolirte Schulterlähmungen nicht erst von Erb gesehen und beschrieben, aber auch nicht zuerst 2 Jahre zuror von Duchenne. Den Chirurgen sind sie längst bekannt, z. B. bei Oberarmverrenkungen. Beispielsweise erwälnt schon Wernber 1855 in seiner Chirurgie (Bd. III a. S. 70), dass dabei Lähmungen bald in einzelnen Muskeln, besonders dem Deltoides, bald im ganzen Arm als Complication vorkommen. Sogar schon Stromeyer kennt 1844 diese zwei Arten von Lähmungen (Chirurgie. I. S. 629) nach Verrenkung des Oberarms und erwähnt, dass die Ableitung dieser Deltoideslähmung von einer Zerreissung des Nervus circumflexus zwar hergeleitet werde, aber nicht erwiesen sei. Uebrigens hat er „Paralysen des Deltoides und der übrigen Muskeln, welche am Schulterblatt entspringen und den Oberarm bewegen, gar nicht selten bei kleinen Kindern in der Zahnperiode entstehen sehen. Während die Bewegungen der Hand und des Ellenbogens fortdauern, hört das Kind auf, den Oberarm zu bewegen, ohne dass man an demselben Geschwulst oder Empfindlichkeit bei Druck wahrnimmt". Diese Lähmungen fand er oft heilbar, ohne dass sich daraus erst das gewöhnliche Schlottergelenk entwickelt, wie ich es auch friher in meiner Arbeit über "Die Mechanik des Huiftgelenks" ${ }^{1}$ ) beschrieben habe. Immerhin sind nach dem, was ich gesehen, in der Chirurgie diese isolirten Lähmungen verschwindend selten gegenüber den gewöhnlichen Nervenverletzungen am Arm.

Erb gebührt das Verdienst, zuerst eine anatomische Erklärung aufgestellt und durch den "Erb'schen Pankt" unterstïtzt zu haben; daher das Interesse für die "Er'b'sche Wurzellähmung “. Mit Aufstellung der Erb'schen Wurzellähmung wurde eine ganze Literatur erweckt!2)

vorderen Fläche der Wirbelsäule bei möglichst starker Adduction der Schulter, ungefähr in einer Linie rom Sternoclaviculargelenk zum Proc. spin. des 7 Halswirbels, etwas nach vorn, $11 / 2 \mathrm{Cm}$. vom Rande des Musc. cucullaris

1) Reichert's und Dubois-Reymond's Archiv. 1965. Heft V.

2) Man vergleiche:

Erb, „Ueber eine eigenthümliche Localisation der Lähmungen im Plexus brachialis" in Verhandlungen des Heidelberger naturhistor. medic. Vereins rom 18. November 1974. S. 130.

Erb, Handbuch der Elektrotherapie. 1952. I. S. 295. 


\section{Unser Fall ist folgender:}

Der Tuchmacher Karl Gerber aus Kottbus, ein mässig kräftiger Mann von 46 Jahren mit normalen Brustorganen ohne Albuminurie oder sonstige Krankheit und ohne hereditäre Belastung, liess sich am 8. Februar 1886 in Bethanien (Journ. 332, Saal 75) wegen "Drüsen am Halse " aufnehmen, die ihm beschwerlich fielen. Weder in der Anamnese, noch bei der Untersuchung ergaben sich Anhaltspunkte für Lues, seine Geschwulst am linken

Dr. H. ten Cate Hoedemaker, „Ueber die von Erb zuerst beschriebene combinirte Lähmungsform an der oberen Extremität" in Archiv für Psychiatrie und Nervenkrankheiten, 1879. Bd. IX. Heft 3. S. 738 .

Bernhardt, "Ueber isolirte Armnerven und combinirte Schulterarmlähmungen" im Centralblatt für Nervenheilkunde, 1854. Nr. 22.

Derselbe, "Beitrag zur Lebre von den Lähmungen im Bereich des Plexus brachialis." Zeitschrift für rat. Medicin. 1882 . S. $\$ 15$.

H. Secretan, Contribution à l'étude des paralysies radiculaires du plexus brachial. Thèses de Paris 1885 .

1I. Larrade, Paralysie spontanée du plexus brachial. Thèses de Paris 1850.

Strauss, Note sur un cas de paralysie spontanée du plexus brachial (avec integrité du nerf médian) et sur quelques localisatious de paralysies du plexus brachial. Gaz. hebdom. 1850.

Depaul, Paralysie du bras chez un nouveau-né extrait à l'aide du forceps. Gaz. des hôp. 1867. p. 90 .

E. Dufourt, Note sur un cas de paralysie radiculaire spontanée du plexus brachial in Lyon médical. I\$\$6. Vol. 31. No. 4. p. 107.

Féré, Etude anatomique et critique sur les plexus des nerfs spinaux in Arch. de neurolog. 1883. Mars. No. 15.

David Ferrier and Gerald Yeo, The fonctionnal relations of the motor roots of the Brachial et Lombosacral plexuses in Proceedings of the Royal Society. 1851. March 24. Juni 16. Vol. 32. p. 12.

Forgues, Distribution des racines motrices dans les muscules des membres. Th. de Montpellier. 1883.

Remak, Zur Pathogenese der Bleilähmungen in Archiv für Psych, und Nerveuheilkunde. 1875. Bd. VI. Heft 1.

M1te. A. Klumpke, Contribution à l'étude des paralysies radiculaires du plexus brachial. Paralysies radiculaires totales. Paralysies radiculaires inférieures. De la participation des filets sympathiques oculo-pupillaires dans ces paralysies in Rev. de médecine. Paris 1885 . No. T. p. 59 L.

J. L. Prevost, Des paralysies radiculaires in Revue médic. Genève. 1556. p. 211.

J. Ross, Rupture of the Brachial Plexus in A Treatise of the Diseases of the Nervous System. 2 Ed. 1883 . Vol. 1. p. 631.

Sands and Seguin, A case of traumatic brachial neuralgia treated by excision of the cords which go to form the brachial plexus in Arch. of scientific. and pract. med. isi3. Vol. I. Jan. No.1.

Ernst Remak, "Zur Pathologie der Lähmungen des Plexus brachialis." Berliner klin. Wochenschrift. 1877 . Nr. 9. S. 116.

Duchenne, L'electrisation localisée. III. Ed. p. 420 u. 357.1872 über die Entbindungslähmungen.

Erb, Krankheiten der peripheren cerebrospinalen Nerven in Ziemssen's Handbuch. Bd. XII. I. 1574. S 510.

F. Franck, Recherches sur les nerfs dilatateurs de la pupille. Trav. du laborat. du prof, Marey. $1875-1879$. p. 26.

Derselbe, Recherches anat. et expér. sur le nerf vertébral. Soc. de biol. 1875. p. 140.

Derselbe, Article Grand Sympathique du Dictionn. Encyclop. des Scienc. méd. III. Sér. XIV. p. 66.

Schiff's Vorlesungen, gesammelt von P. Marchi (Florence 1573. XVII).

Guillebean und Luchsinger, „Existiren im Nervus vertebralis wirklich pupillendilatirende Fasern?" in Pflüger's Arch. f. ges. Physiol. Bd. XXII. 1880. S. 156 und andere mehr. 
Halse ist eine isolirte; insbesondere finden sich keine Drttsen in ihrer Umgebung oder in der linken Achsel. Sie sei erst seit 6 Wochen bemerkt, obgleich sehr intensive Schmerzen schon seit dem Januar 1885 im linken Arm bestanden. Einmal sei der Arm ,trocken " gewesen, aber durch Bäder und Elektrisiren wieder gebessert worden. Seit diesen 6 Wochen hätten sich mit dem Anwachsen der Geschwulst auch die Schmerzen verstärkt.

Sie liegt im linken Trigonum cervicale posticum in der Längsrichtung des Halses gleich unter der durch Jodanstrich infiltrirten Haut, ist ans mehreren wallnussgrossen Knollen verbunden, ziemlich glatt, prall-elastisch und erstreckt sich mit dem unteren Ende etwas unter das Schlüsselbein, während das obere hinter dem Kopfnicker verschwindet. Mitbewegung beim Athmen und Schlucken wie beim Kropf lässt sich nicht bemerken. Die Geschwulst lässt sich auf ihrer Unterlage verschieben und ein Zusammenhang mit den an sich nicht empfindlichen Halswirbeln nicht bemerken. Die Hant dariber ist verschieblich, niclıt sehmerzhaft und sonst nicht verändert; dagegen schmerzt die Geschwulst an ihrem unteren Ende auf Berührung besonders stark. Der Arm ist nicht verändert, es findet sich kein Zeichen von Lähmung; nur ist links wie oft der Händedruck etwas schwächer als rechts. Fieber bestelit nicht, jedoch schlaift der Kranke der Schmerzen wegen die Nacht nicht trotz 2 Pulvern mit 0,01 Morph. hydrocliloratum.

Bei der Operation am 10. Februar machte ich unter Carbolspray auf der Geschwulst einen Längsschnitt und fast senkrecht dazu an seinem unteren Eude einen Querschnitt nach vorn und hinten dicht oberhalb des Schlïsselbeins wie bei der Ligatur der Subclavia. Nach Durchschneidung der infiltrirten Haut und des Hautmuskels präparirte ich die Lappen möglichst weit zurück, durchschnitt den M. omoliyoides und überzeugte mich beim vorsichtigen Herauslösen der wurstförmigen, glatten, grawen Geschwulst, dass sie oben und unten in der Tiefe festsass. Nach seitlichem Ablösen des Musc. scalenus auticus und Aufheben seines Aussenrandes mittelst eines gabeligen Muskelhakens fand ich, dass die sich hinter dem Muskel scharf absetzende Geschwulst in zwei etwa bleistiftdicke Nerven von gewöhnlichem Ausselien iberging; der Lage nach waren es der vordere Ast des 5. und des 6 . linken Halsnerven. Ilure Durchtrennung bei stärker emporgehobenem Musc. scalenus anticus gelang etwa $: 3 / 4 \mathrm{Cm}$. oberhalb der Gescliwulst; es erfolgte dabei ein so starker Ruck im Arm, dass es selbst mir beim Durchschneiden auffiel. Der Arm wurde neben dem Körper wie bei der Lnterbindung der Subclavia festgehalten; indem ich ihn in dieser Richtung möglichst stark herabziehen liess, gelang es, das untere mehr spitze Ende der Geschwulst mit zwei austretenden Nerven oberhalb des Schlüsselbeins zu Tage zu fördern; ihre Durchtrennung erzeugte ebenfalls einen kräftigen Ruck im Arm. Die iibrigen Nerven am Halse wurden nicht blossgelegt; seitliche Verbindungsäste des Nerventumor und seiner 4 Füsse liessen sich nicht bemerken, brauchten also auch nicht durchtrennt za werden.

Die Blutung war nicht von Belang; nachdem vier Drains in die drei Ecken der $\mathrm{T}$-förmigen Wunde und in die Berührungsstelle der zwei Schnitte angelegt, wurde die Wunde genäht und antiseptisch verbunden. Am folgenden Tage (den 11. Februar) fand sich bei der Visite nichts mehr von den Schmerzen vor, alch war keine Störung an dem jm Verbande freigelassenen Vorderarm zu bemerken. Er konnte die Finger gut bewegen und 
füblte damit; Pro- und Supination war unverändert. Einige Male klagte der Kranke anfangs noch beim Verband über Reissen im Arm, was sich jedoch bald verlor. Die Wunde heilte trotz der Reste des Jodanstrichs durch Verklebung ohne Fieber oder irgend einen sonstigen Zufall. Die letzte Drainstelle war eben überhäutet und mit einem leichten Schutzverband bedeckt (am 5. März), als der Kranke plötzlich aus äusseren Gründen wider Erwarten nach Hause reisen musste.

Der Erfolg der Operation in Bezug auf die Nervenläbmung wurde von mir bei Gelegenheit der antiseptischen Verbände constatirt; Gerber musste, um seinen linken Arm im Bett zu gebrauchen, ihn mit der rechten Hand dahin legen, wo es nöthig war. Dort konnte er dann mit der Hand machen, was er wollte, wie zuvor. Die Bewegung der Hand und Finger war links gerade so gut als rechts. Eine Anästhesie lässt sich nirgends an der ganzen linken Oberextremität nachweisen, auch lässt sich kaum ein Unterschied in der Stärke des Händedrucks wabrnehmen. Die Vorderarme lagen dabei parallel auf den Knien im Bett, die Hände neben den Ellenbogen; auch in der Rotation der Hände, dem Umfang der Pro- und Supination, liess sich dann keine Differenz bemerken. Im Bett, konnte es scheinen, fehle jede Lähmung. Anders beim Stehen! Jede Herrschaft iiber den Oberarm war verloren; wie ein Pendel hing er am Körper herab. So vollständig und leicht sich passiv jedes Gelenk bewegen liess, activ ribrte sich der Oberarm nach keiner Richtung eine Spur. Beim Herabbängen des Arms war der Rotationskreis der Hand links etwas geringer als rechts, indem dabei sichtlich (an den Condylen des $\mathrm{Hu}$ merus) die Dreher des Oberarms im Schultergelenk versagten. Das Achselzucken ging auf beiden Seiten gleich gut, wie auch Erscheinungen von Seiten des Halses und Kopfes nicht zu bemerken waren.

Von seinen Schmerzen befreit, verliess uns der Kranke zufriedener, als ich nach der Grösse der Verletzung erwartet hatte. Welch Unterschied gegen die nns so gewöhnlichen Lähmungen an den Fingern! Der Kranke aus der Romberg'schen Praxis, dem Wilms in meiner Assistentenzeit den Ulnaris wegen eines Myxoms ${ }^{1}$ ) am Oberarm excidirte, bekam schon vor der Entlassung zu seiner Ulnarisparalyse eine von der kleinen Daumenmusculatur beginnende und sich

1) Virchow hat es in seiner Geschwulstlehre. Bd. I. S. 426 in Fig. 85 abgebildet. Wie ich schon in meiner Arbeit über das Del. trem. und traumaticum (S. is) erwähnt habe, kam es in diesem Fall zu einem schweren Erysipelas phlegmonosum haemorrhagicum mit vielen Incisionen, so dass man hier vielleicht noch Bedenken tragen konnte, die Atrophie nach Art der Aran'schen Atrophie auf die Excision des Neuroms zu beziehen. 
quer gegen den kleinen Finger verbreitende Atrophie der Handmuskeln; dass die trophischen Fasern der kleinen Handmuskeln in der That im Ulnaris liegen, habe ich seitdem oftmals bei einer Verletzung am Vorderarm erlebt. Wie unglicklich, wie unzufrieden ist der Kranke, wenn selbst der Armnerv leidet, der doch von allen drei auf das kleinste Gebiet an den Fingern begrenzt ist, der N. ulnaris. Und bier waren am Halse zwei viel dickere Nervenstämme excidirt, und der Kranke nicht zu halten! Eine Aufforderung, an den Fingern die conservative Chirurgie bis zum Aeussersten zu treiben!

Bei einer Nachuntersuchung am 6. Juni - der Kranke hatte sich die ganze Zeit 3 mal täglich 10 Minuten lang den Arm selbst faradisirt - ergab sich über das definitive Resultat, die Folgen und Ausgleichungen Folgendes:

Der horizontale Theil der Narbe ist etwa $10 \mathrm{Cm}$. lang und sein Aussenende durch die verticale $7 \mathrm{Cm}$. lange Narbe etwas hinaufgezogen; durch eine leichte Contractur der Platysmafasern in der Längsnarbe springt dieselbe bei starker Drehung des Kopfes nach rechts etwas vor und bewirkt dadurch zuletzt das Gefühl von Spannung.

Trotz des Elektrisirens ist an einigen Stellen Atrophie eingetreten; der Musc. deltoides ist abgeflacht, so dass der Kopf des Oberarmes deutlicher durchscheint. Die Ober- und Untergrätengrube sind infolge Atrophie der Mm. supra- und infraspinati etwas eingesunken. Die Zackenansätze des Musc. serratus ant. major sind selbst bei der Ruhelage rechts sebr deutlich, links nicht zu sehen, ebensowenig wie der Muskel selbst. Die ganze Oberarmmusculatur ist abgemagert, dagegen bat die Musculatur am Pect. major und Latiss. dorsi eber zugenommen. Das Gefühl am Arm hat nirgends gelitten, nur sind passive Bewegungen im Schultergelenk, welches durch die theilweise Lähmung etwas zu wenig bewegt wird, bei starker Abbewegung des Oberarmes, z. B. beim Legen der Hand auf den Kopf, zuletzt empfindlich; ein Schlottergelenk hat sich bis jetzt nicht ausgebildet.

Was die active Bewegungsfähigkeit betrifft, so bleibt beim Versuch, sich an die Nase zu fassen, der Arm ganz gestreckt, wird dagegen bis zu einem Winkel von etwa $30^{\circ}$ in der Sagittalrichtung erhoben, wobei das Schulterdach kräftig durch den M. cucullaris gehoben und nach oben fixirt, der Pect. major stark angespannt wird.

Beim Versuch, in der Querrichtung seitlich den Arm zu erheben, bewegt sich nichts im Musc. deltoides noch M. serrat. anticus major, dagegen gelingt es dem Kranken, den gestreckten Arm seitlich vom Leib (bis zu einem Winkel von $30^{\prime \prime}$ etwa) abzuschleudern; im nächsten Moment fällt er zurtick und kann dauernd vom Leibe etwa in 
einem Winkel von $5^{0}$ abgehalten werden, das jedoch nur, indem das Schulterdach dabei vom Rande des M. cucullaris kräftig in die Höhe gehoben und so etwas mit dem äusseren Ende emporgedreht wird.

Nach hinten kann der Arm in der Sagittalrichtung nicht soweit bewegt werden als der rechte. Rechts geschieht es bis zu einem Winkel von $60^{\circ}$, links bis $30^{\prime \prime}$ circa mit Spannung des Latissimus dorsi und Triceps.

Betrachtet man den Kranken von hinten, so kann er beide Sehulterblätter ganz gleichmässig einander nähern, nach oben und nach aussen ziehen.

Legt man die Hand in seine linke Achsel, so kann er recht krättig sie zwischen Leib und gestrecktem Arm einklemmen, wobei man fühlt, wie sich mächtig die Muskeln am unteren Rande der Achselgrube hinten und rorn spannen, wie uberhaupt die M. cucullaris, latissimus dors. und pectoralis major durch die Uebung an Kraft zugenommen haben. Mittelst dieser Fixirung des Schultergelenks beherrscht also der Kranke etwas den Oberarm, der sich mit dem Schulterdach mitbewegt.

Wenn er beim Sitzen die Ellenbogen auf den Oberschenkel aufstemmt und die Arme darauf mit der Volarseite ruben lässt, so ist gar kein Unterschied zu bemerken bei Bewegung der Hände; legt er die Riickseite der Vorderarme auf die Oberschenkel, so erfolgt links die Beugung der Hand nicht so vollkommen als rechts; links bis zu einem Winkel von $40^{\prime \prime}$, rechts bis $z \mathfrak{u} 80^{\circ}$ etwa. Dabei dreht sich links gern die Hand, so dass der Daumen, nach einwärts rotirend, umfällt.

Während bei rechtwinkliger Haltung des Ellenbogens und Aufruhen des Vorderarms Pro- und Supination der Hand von beiden Seiten einen gleichen Kotationskreis hervorruft, ist der Umfang der Rotation am hängenden Arm verschieden. Die Beobachtung ist freilich dadurch etwas erschwert, dass die Arme bei der Streckung etwas an der radialen Seite im Ellenbug stumpfwinklig gebengt sind, nach Art des Genu valgum. Es können dabei beide Daumen einwärts soweit gedreht werden, dass sie sagittal nach hinten gerichtet sind bei Berührung des Leibes durch den Handrücken, freilich zittert dabei zuletzt der linke Arm. Von dieser Stellung aus kann der rechte Daumen wie gewöhnlich um vier rechte Winkel nach anssen gedreht werden, bis der Hand- und Vorderarmrïcken wieder den Leib beriihrt, der linke jedoch nur etwa um 21/2 rechte Winkel. Dabei macht! dentlich der Condylus internus humeri um die Armaxe links eine Drehung von ungefähr 1-2 Drittel eines rechten Winkel mit. Rechts kommt der vierte rechte Winkel nur anf besondere Aufforderung zu Stande, links 
um keinen Preis. Im Anfang dieser Drehbewegung stehen beide Condyli interni humeri gleichmässig nach hinten aussen. Wenn man beim Hängen beider Arme von hinten ihre vier Condyli festzuhalten versucht, was bei der Muskelstärke sehr schwer ist, so schien die $\mathrm{R}_{0}$ tation links einen, rechts zwei rechte Winkel zu betragen.

Ob der Triceps eigentlich gelähmt ist? Atropbisch ist er jedenfalls sehr. Eine Untersuchung mit dem Inductionsapparat zeigt, dass sich der flache Muskel oberhalb des Olekranonansatzes deutlich und straff spannt bei der elektrischen Erregung, wie man sich dann davon auch bei willkürlicher Erregung überzeugen konnte. Trotzdem der Strom recht unangenehm stark gemacht werden konnte, gelang es nicht, damit eine Beugebewegung im Ellenbogen hervorzurufen. Setzte man einen Schwamm auf vorn oberhalb des Schltisselbeins und den anderen am Oberarm, so streckte sich straff der Triceps, die Hand wurde flectirt und der Daumen schlug nach innen, selbst wenn man den zweiten Schwamm auf den Biceps setzte. Die Muskeln an der Vorderseite des Oberarms liessen sich anscbeinend nicht zur Contraction bringen, ebensowenig Supra- und Infraspinatus, Deltoides, Serratus anticus major. Dagegen sind Pectoralis major, minor, Rhomboidei, Cucullaris, Latissimus dorsi sehr kräftig.

Was endlich die Gebrauchsfähigkeit des Arms betrifft, so war es interessant zuzuseben, wie sich der Kranke obne Hulfe sein Reisecostüm aus- und anzog. Der linke Triceps schien nur dazu zu dienen, den linken Arm steif zu machen. Brauchte er ihn nicht, so legte er mit der rechten Hand die linke im Stehen auf den Tisch mit der Hohlhand auf, wie um sie los zu sein. Uebrigens knöpfte er sich die Knöpfe seiner rechten Manschette mit der linken Hand zu. Nach seiner Angabe arbeite er seit 14 Tagen; es gehe ganz gut, wenn er einen Ruhepunkt habe.

Alle diese Erscheinungen lassen sich wohl in folgender Weise deuten. Da der Arm weder seitlich erhoben, noch der Ellenbug gebeugt werden kann, sind gelähmt M. deltoides und Serratus anticus major, ferner M. biceps, brachialis internus, coracobrachialis, supinator longus, welche auch hinterher sichtlich abmagerten und elektrisch nicht mehr erregbar sind. Pro- und Supination waren bei rechtwinkliger Lage normal; die Pronatoren und Supinator brevis sind also ebenso unbetheiligt, wie die übrigen Hand- und Fingermuskeln. Wenn der Rotationskreis beim Hängen des Arms um ein Drittel rechten Winkel grösser war, als bei jener Lage, so rührte das wohl von der Mitbetheiligung des Latissimus dorsi her, der, jetzt besonders 
gekräftigt, soweit die Pronation durch Rotation des Humerus verstärkte; denn Biceps und die Aussenroller (Supraspinatus, Infraspinatus, Teres minor) des Oberarms sind nicht blos gelähmt, sondern jetzt auch abgemagert. Die anderen Innenroller, Teres major und Subscapularis werden wohl auch gelähmt sein, sonst mlisste, denk' ich, beim Hängen des Arms der Unterschied der Rotation noch grösser sein gegenüber der Rotation bei aufgestemmtem Ellenbogen. Secundär sind trotz allen Elektrisirens atroph sichtlich M. supraspinatus, infraspinatus, deltoides, serratus anticus major, biceps, brachialis internus, kräftiger geworden als Ersatzmuskeln Pectoralis major, Cucullaris und Latissimus dorsi, nicht gelähmt, aber durch Nichtgebrauch schwach geworden ist Musculus triceps, weil er anscheinend nur zum Steifmachen des ein- für allemal hängenden Arms benutzt wird. Hervorzuheben ist, dass Supinator brevis nicht gelähmt, dagegen Serratus anticus major, Supraspinatus und Infraspinatus gelähmt sind, gegentiber anderen Beobachtungen.

Alles das ist die Folge der Excision einer Nervengeschwulst, welche von dem gemeinsamen Stamme ausgegangen ist, den der vordere Ast des 5. Halsnerven und der vordere dst des 6 . Halsnerven in dem oberhalb des Schlisselbeins gelegenen Theil des Plexus brachialis bildet; diese beiden oberen Wurzeln, die Erb'schen Plexuswurzeln, wurden dabei mit entfernt.

Es waren also mit der Excision der oberen Erb'schen Plexuswurzeln bei meinem Kranken gelähmt einmal von den 5 Brustkastennerven

gar nicht der N. dorsalis scapulae, da die M. rhomboidei sichtlich nicht gelitten hatten;

deutlich der N. thoracicus posterior, da der Musc. scalenus anticus ganz gelähmt und jetzt abgemagert ist;

gar nicht die Nn. thoracici anteriores, welche sich in die Musculi pectorales, Brustdrise und Haut verbreiten, wohl aber der, welcher sich von innen an den vorderen Theil des M. deltoides begiebt; vollständig der N. suprascapularis, da sowohl M. supraspinatus als auch M. infraspin. vollständig gelähmt und jetzt atrophisch sind; vermutblich die zwei kleineren Nn. subseapulares, da auch Musc. subscapularis und M. teres major gelähmt sein werden. Der N. subscapularis longus ftir den M. latissimus 'dorsi ist nicht gelähmt.

Von den 7 Armnerven sind

unbetheiligt naturlich der $\mathrm{N}$. cutaneus brachii internus, welcher aus den unteren Plexuswurzeln, dem 8. Hals- und 1. Rückennerven stammt; 
unbetheiligt der $\mathrm{N}$. cutaneus brachii medius, der auch hauptsächlich aus den 1. Rückennerv kommt und in die Haut sich ebenso verbieitet;

vollständig vom N. musculo-cutaneus alle Muskeläste, welche Biceps,

Coracobrachialis und Brachialis internus versorgen, gelähmt; vollständig vom $\mathrm{N}$. circumflexus humeri alle Muskeläste, welche den Teres minor, Subscapularis, Teres major und Deltoides versorgen, betheiligt (die Hautäste sind frei);

unbetheiligt der N. medianus;

unbetheiligt der $\mathrm{N}$. ulnaris, und schliesslich

betheiligt vom N. radialis nur die Muskeläste, welche zum Supinator longus und Brachialis internus gehen.

So sehr das Bild unseres Kranken im Allgemeinen also mit den Ansichten von der Erb'schen Wurzellähmung stimmt, ebenso weicht es jedoch wie gewöhnlich im Einzelnen ab.

Es fällt auf, dass der $\mathrm{N}$. dorsalis scapulae unbetheiligt war, der gewöbnlich vom 5. und 6. Halsnerven hergeleitet wird. Die Nn. thoracici anteriores werden ebenso meist von ihnen gebildet, sind jedoch, mit Ausnahme ibrer Aeste für den Deltoides, auch nicht gelähmt.

Von den Armnerven sind allein N. musculo-cutaneus und circumflexus humeri mit allen ihren Muskelästen, der $\mathrm{N}$. radialis mit den beiden Muskelästen für Supinator longus und Brachialis internus bei unserem Gelähmten nach gewöhnlicher Annahme betheiligt.

Es entspricht das im Allgemeinen vielleicht dem, was Féré jüngst bei seinen anatomischen Untersuchungen gefunden hat. Ibm zu Folge liefert jedes Halsnervenpaar mehreren Armnerven in variabler Zahl Aeste, und jeder Armnerv erbält von mehreren Halsnervenpaaren in individuell verschiedener Weise Aeste. Nach Féré versorgt insbesondere das 5 . und 6 . Halsnervenpaar durch den $\mathrm{N}$. musculo-cutaneus die Mm. biceps, brachialis internus, coracobrachialis, durch den N. circumflexus den Musc. deltoides und giebt die Nerven für den M. supra- und infraspinatus, teres major, latissimus dorsi, pectoralis major, serratus anticus major und supinator longus. In unserem Fall sind jedoch Latissimus dorsi und Pectoralis major nicht gelähmt, sondern übermässig kräftig in Function. Dagegen wird der Nachweis Fèré's, dass die Radialisfasern für den Supinator longus sich bis in die 6 . Wurzel verfolgen lassen, durch unseren Fall bestätigt, indem hier jede Spur von Beugung im Ellenbogen fehlte bei Excision der Wurzeln vom 5. und 6. Halsnerven. 
Es haben dann die Verbreitung der 5. und 6. Wurzel studirt Ferrier und $\mathrm{Ye}_{\mathrm{o}}$ bei Affen, indem sie nach Eröffnung des Wirbelkanals unmittelbar die vorderen Wurzeln reizten. Sie kamen zu dem Resultat, dass jede einzelne vordere Wurzel eine bestimmte combinirte Function einer Muskelgruppe besorge, ibre Durchschneidung ein gewisses Zusammenwirken einer solchen Gruppe aufhebe; indem jeder Muskel Aeste von verschiedenen Nervenwurzeln erhalte, spiele er in verschiedenen Muskelfunctionen mit und werde also dann erst vollständig gelähmt, wenn alle diese Wurzeln durchschnitten sind.

Gegenuiber dieser "Systématisation fonctionnelle" wiirde zu betonen sein, dass hier schon bei Durchschneidung von zwei Wurzeln beim Menschen eine ganze Reihe Muskeln für jede Function vollständig sofort gelähmt wurde, z. B. die Aufheber und Auswärtsroller des Arms, die Beuger des Vorderarms (Deltoides, Serratus antic. major, Biceps, Brachialis intern., Supra- und Infraspinatus).

Insbesondere soll es nach Ferrier's und $\mathrm{Yeo}^{\prime}$ 's Versuchen die Function der 5. Wurzel sein, die Hand zum Munde zu bewegen; sie stände also vor der Erhebung einwärts und Beugung des Vorderarms mit Supination und Extension der Hand und Finger (dem Deltoides, Biceps, Brachialis internus, Serratus anticus major, Supinator longus, Extensor carpi und digitorum). Diese Versuche am Affen stimmen insofern nicht mit unserem Fall, als hier das Führen der Hand zum Munde zwar unmöglich gemacht, die Erhebung und Beugung des Arms vollständig aufgehoben, die Extension der Hand und Finger aber gar nicht betheiligt war.

Forgues erzielte in seinen Experimenten, welche er an Hunden im Laboratorium von Lannegrace in Montpellier machte, bei der Durchscbneidung der Plexuswurzeln keine sicheren Resultate. Er zog es dieser Methode vor, die Wurzeln bei dem Austritt ans dem Ruickgrat erst zu durchschneiden und dann zu reizen. Nach seinen Versuchen am Affen gelte die physiologische Specialisation in Wahrheit nur für die Endstämme der Nerven, z. B. Medianus, Radialis. Jede Wurzel stehe nicht einer combinirten Function, sondern einem topographisch constanten Muskeldepartement vor. Jede Wurzel versorge beide Seiten der Gliedmassen. Je tiefer abwärts sie liege, desto mehr würden die unteren Abschnitte der Glieder betroffen. Je mebr die Reizung den Rückenwurzeln sich nähere, desto mehr erstreckten sich die Contractionen von den Muskelmassen der Radialseite zu der der Ulnarseite.

Dieses letzte Gesetz kann ich insofern umgekehrt für den Menschen 
bestätigen, als ich mehrfach gesehen habe, dass bei schnell im Rückenmark aufwärts steigenden Lähmungen, wenn die oberen Glieder ergriffen wurden, zuerst am Arm und manchmal allein das Gebiet der Nn. ulnares gelähmt wurde. Unter mehr als 100 Wirbelbrïchen, die ich behandelt habe, waren mehrere mit Querlähmung der Beine nach Zerreissung des Rückenmarks, welche nach 8 Tagen ungefähr an Blutung in den Rückgratskanal, wie die Section zeigte, starben. Wenn sich dabei durch Zunahme des Druckes die Lähmung nach oben verbreitete, so wurde zuerst das Ulnargebiet gelähmt, und blieb es oft bis zum Tode allein.

Wenn dagegen Forgues von den Einbriichen in diese Gesetze bemerkt, dass die 6. Wurzel vom Deltoides gleich zum Supinator longus und den Radiales spränge, ohne Teres major, Latissimus dorsi und Triceps zu betheiligen, wie andererseits die 8. Wurzel mit Uebergehung ibres Vorderarmbezirks den Pectoralis major, Teres major und Latissimus dorsi versorge, so stimmt das mit unserer Erfahrung am Menschen wieder insofern nicht ganz, als ja hier die Mm. radiales ganz unbetheiligt geblieben sind.

Frl. Klumpke hat mehrere Fälle von Lähmung der unteren beiden Plexuswurzeln gesammelt, in denen neben der Ulnarislähmung Myosis, Verengung der Lidspalte und Retraction des Bulbus auffielen, ohne dass vasomotorische und thermische Erscheinungen im Gesicht bemerkt wurden.

Als Budge und Waller das Centrum ciliospinale an der Fuge des 2. und 3. Rückenwirbels entdeckten, fanden sie, dass die vorderen Rïckenmarkswurzeln dieselben Erscheinungen auf die Pupille äusserten, wie der Sympathicus. Bernard wies dann nach, dass die $\mathrm{Pu}-$ pillenfasern im Rückenmark getrennt von den vasomotorischen entspringen und auch von ihnen getrennt beim Hunde durch die erste und zweite vordere Rückennervwurzel gehen direct in das Ganglion stellare, während die vasomotorischen durch die vorderen Wurzeln des 3.-6. Rückennerven erst noch den Brustsympathicus durchstreichen. Auch am Halssympathicus lassen sich nach Schiff beide Faserarten bei manchen Individuen sehr leicht scheiden.

Indem der erste Riickennerv sich bei der Bildung der unteren Plexuswurzeln betheiligt, werden bei der unteren Plexuswurzellähmung diese Verhältnisse von Bedeutung.

Nach Franck sollen nun auch die Rami communicantes vom 8., 7. und 6. Halsnerven, ja sogar vom 5. Halsnerven für das erste Ganglion thoracicum Pupillenfasern enthalten. Dem gegenüber hat Frl. 
Klumpke mit Unterstützung von Queyrat im Laboratorium von Vulpian an Hunden gefunden, dass Verletzungen des Plexus brachialis in Wirklichkeit nur dann Pupillenphänomene zeigen, wenn der Ramus communicans des 1. Dorsalnerven dabei mit rerletzt ist; niemals sind zugleich vasomotorische Störungen vorhanden. Der 1. Dorsalnerv ist demnach der oberste, welcher die Pupille beeinflusst. Die vasomotorischen Gesichtsnerven gehen in der That durch den 3. bis 6. Rückennerv. Diese Begrenzung wurde, entgegen der Ansicht von Franck, bestätigt durch die neuen Versuche von Prevost, die er mit P. Binet in Genf an Hunden und Katzen gemacht hat. Indem sie an curarisirten Thieren, welche mittelst kïnstlicher Respiration am Leben gehalten wurden, das Rückenmark blosslegten und Schicht fïr Schicht quer durchschnitten, fanden sie bei elektrischer Reizung der vorderen Wurzeln dies- und jenseits der Querschnitte manchmal schon am 8. Halsnerven, meist aber erst beim 1. und 2. Rückennerren bei Katzen (einmal auch beim Kaninchen noch beim 3 . Rückennerven) ein Hervortreten des Augapfels mit Erweiterung der Pupille, was jedoch niemals bei Reizung des 7 . Halsnerven oder eines höheren eintrat.

Danach kann das Pupillenphänomen (wenn es vorhanden, entsprechend dem bohen Sitz der Lähmung an den Verbindungslöchern selbst am Austritt der Wurzeln) als charakteristisch für die untere Plexuswurzellähmung wohl angesehen werden, bei der der 8 . Halsund 1. Ritckennerv ins Spiel kommen. Bei unserem Kranken war ron der Myosis, der Verengung der Lidspalte und der Retraction des Bulbus nichts zu bemerken, weder während der Heilung, noch auch nachträglich bei der Revision am 6. Jani, obgleich die Plexuswurzeln zwischen den $\mathrm{Mm}$. scalenis selbst zerschnitten waren, so dass die $\mathrm{Mm}$. supra- und infraspinati gelähmt waren, deren Nerv zuhöchst entspringen soll. Es spricht also dieser Fall gegen die Ansicht von Franck für die Uebertragbarkeit der Thierversuche von Bernard, Frl. Klumpke nnd J. L. Prevost auf den Menschen.

Schliesslich noch eine Bemerkung!

Bei einem Fall von reiner, oberer Erb'scher Wurzelläbmung, welche sich ohne Betheiligung der Sensibilität auf den Deltoides, Biceps, Brachialis internus, Infraspinatus, Serratus anticus major, Supinator longus und auch Supinator brevis erstreckte, hat Dufourt eine Abnabme der Schweisserzeugung zur Zeit des Gebrauchs ron Pilocarpin constatirt, woranf bisher dabei nicht geachtet worden zu sein scheint. Unser Kranker hat wegen des antiseptischen Verbandes, 
bei dem noch ein starker Ueberverband mit Salicylwatte Brust, Hals und Oberarm einhïllte, im Krankenhaus mehr geschwitzt, als ihm lieb war, ohne dass man in dieser Beziehung zwischen beiden Körperseiten einen Unterschied bätte wabrnehmen können. Auf Befragen hat er mir erklärt, auch draussen habe er viel bei den heissen Tagen dieses Sommers geschwitzt, ohne einen Unterschied derart zu bemerken.

Jedenfalls wäre der Einfluss der Erb'schen Lälumung auf das Schwitzen also kein constantes Zeichen. Dafür spricht auch, dass Sands und Seguin bei ibrer Durchschneidung aller 5 Plexuswurzeln am Menschen sofort Myosis und Vermehrung der Schweisssecretion auf der betreffenden Seite gerade umgekehrt baben eintreten seben, während andererseits Ross und Frl. Klumpke bei ibren Fällen von totaler Plexuslähmung neben Myosis und Verengung der Lidspalte den Arm trocken saben, wenn der ganze Körper sonst dureh Pilocarpin reichlich schwitzte. Bei den übrigen Fällen, Frl. Klumpke hat eine ganze Zahl gesammelt, scheint man auch hier nicht weiter darauf geachtet zu haben.

Nachdem ich im Bisherigen unseren Fall von Excision der Erbschen Plexuswurzeln in physiologischer Beziehung mit den vorhandenen Fällen und Thierversuchen, soweit sie mir bekannt, verglichen habe, eriibrigt es sich wobl noch, in chirurgischer Beziehung auf mein Vorgehen bei der Operation zurückzukommen. Einer Rechtfertigung bedarf es wohl nicht, da ich denselben Weg eingeschlagen habe, den ich allein von anderen Chirurgen habe ausführen seben. Ich erinnere blos an den angeführten Romberg'schen, von Wilms in Bethanien operirten und von Virchow abgebildeten Fall eines Neuromyxoms des Ulnaris.

Allein in meiner Zürcher Zeit habe ich grundsätzlich anders, conservativer gehandelt; da jedoch mein junger Urner Assistent, dem ich vor meinem Abgang alle meine Neuromoperationen zur Bearbeitung ibertragen habe, leider eben unerwartet den Anstrengungen seiner Thätigkeit im Gebirge in aller Jugend erlegen ist, will ich hier in Kürze darüber Bericht erstatten. Bei diesen conservativen Bestrebungen handelt es sich natitrlich nicht um Nervenastgeschwülste, noch um Nervenstumpfgeschwïlste, bei denen sie wenig Werth hätten, sondern um die isolirten Nervenstammgeschwïlste. ${ }^{\text {) }}$

1) In den 14 Jahren meiner Klinik in Zürich haben sich nur drei Nervenastneurome operiren lassen. 1872 wurde eine Frau mit einem Neurom unter der Haut 
Für den Operateur zerfallen meines Erachtens alle mit einem Nervenstamm verbundenen Geschwülste in drei Klassen, die ich ,anbaftende ", "eingebettete " und "durchwachsene " nennen möchte. Letztere, bei denen sich die Züge der Nervenfasern nicht mehr isoliren lassen, schliessen sich hier von selbst aus.

Bei den „anhaftenden" Neuromen hängt die Geschwulst nur an einem Theil des Umfangs mit dem Nerv zusammen, und zwar inniger als mit der Nachbarschaft sonst, so dass man eben bei gutartigen einen Grund hat, überhaupt an eine Entstehung ans den Bestandtheilen des Nervenstammes zu glauben. Im Einzelfall kann sich der Zusammenhang auch hier sehr verschieden gestalten in allen Uebergängen zwischen dem Vorkommniss, dass eine kugelige Geschwulst einen cylindrischen Stamm blos berührt, oder dass im Gegensatz ein Nervenstamm handlang der ganzen Länge einer Gesehwulst anliegt, entweder vorspringend über ihre Fläche oder in ganzer Länge seines Verlaufs in einen Graben der Geschwulst versenkt. In beiden Fällen liegt es nahe, die Geschwulst mit Erhaltung des Nervenstammes zu entfernen, und braucht man sich dadurch nicht stören zu lassen, dass voraussichtlich und nachher in der That der Nerv durch die Wundböhle geschlängelt verlaufen muss, weil er mit dem Wachsthum der Geschwulst allmählich in der Länge hat mitwachsen müssen; besonders wird sich der Nerv dabei verlängern, wenn die Geschwulst unter ihm noch Buckel bildet. Eine Verdiunnung des Nervenstammes babe ich dabei nicht beobachtet, und brauchen auch bei weichen, langsam wachsenden Geschwiilsten Druck und andere Erscheinungen von Seiten des Nerven nicht einzutreten. Der Nerv wächst hier in verstärktem Maasse, wie die Tunica albuginea beim Carcinoma testis.

Einen solchen Fall bot mir ein handgrosses fibröses Lipom ohne Lappen in der Ellenbeuge, welches eng mit dem darauf liegenden Medianus verwachsen war und der Gelenkkapsel locker auflag. Er war zu einer Schlinge verlängert und ging vor der Geschwulst, welche

des Rückens und eine zweite mit einem Neurom der Haut des Unterschenkels geheilt entlassen. 1875 exstirpirte ich bei einer jungen Person eine mir nicht ganz klare flache Geschwulst in der Falte zwischen Gesäss und Oberschenkel, die sich nacb der Untersuchung von Hrn. Prof. Eberth als Rankenneurom auswies; leider starb die Person im Verlauf der Heilung an einem ganz unerwarteten Abort. Díe übrigen Fälle betrafen Nervenstammgeschwülste, im operativen Sinne gesprochen, wonach uns jede Geschwulst doppelt interessirt, die mit einem Nerven zusammenbängt; "wahre Neurome" im pathologisch-anatomischen Sinne waren nicht darunter. Im Ganzen waren es 11, mit den 3 Nervenastneuromen 14 in 14 Jahren. In der Sprechstunde habe ich ausserdem noch eine ganze Zahl gesehen, die sich zu einem Eingriff nicht entschliessen konnten. 
sich mit Bildung einer Rinne von ihm abpräpariren liess, so entlang, dass er leider etwa handlang von allen Seiten freigelegt werden musste. Er war jedenfalls unverdünnt, nicht fächerförmig gespalten und lief nach der Operation frei durch eine grosse Höhle, in der die Gefässe und die Gelenkkapsel bloss lagen; der Fall wurde ohne Naht offen behandelt und hinterblieb nach der Heilung kein Nachtheil. Von einem Recidiv wäre mir sicher etwas zu Ohren gekommen; dass es ausblieb, entsprach ganz der Diagnose, die vom Collegen $\mathrm{Eb}$ b rth nach histologischer Untersuchung bestätigt wurde. Die Geschwulst hing mit ihrer Nachbarschaft nur locker, eng dagegen mit dem Nervenstamm in der beschriebenen Weise zusammen, und erinnerte es mich an die seit Meckel bekannten heterologen Hirnlipome, von denen Virchow eine ganze Reibe gesammelt hat. ${ }^{1}$ )

In diesem Fall war das Resultat der conservativen "Ausschälungsmethode" ganz befriedigend, so übel dabei dem Nerven mitgespielt wurde. Es war mir das seiner Zeit damals sehr bemerkenswerth in Hinblick auf meine Bearbeitung des Tetanus, in der ich ihn als Folge localer Nervenreizung auffassen musste. Wenn man in juingster Zeit erfahren, was der Nerv an einmaligem Eingriff ohne Schaden bei der Operation der Nervendehnung aushalten kann, so verliert die Ausschälungsmethode viel von ihrer bedenklichen Seite. Es fragt sich immerhin, ob auch bei bösartigen Geschwülsten der Nerven, die doch in der Mehrzahl uns beschäftigen - von 15 Fällen aus meiner Praxis (abgesehen von den Stumpfneuromen) waren 10 reine oder gemischte Sarkome - die Methode ausführbar ist? Was kommt bei ihr heraus, wenn wir nach dem definitiven Erfolg fragen, und das sollte man doch nach Billroth's nicht genug anzuerkennendem Vorgange heutzutage immer thun!

Für diese operative Frage ist es auch gleichgültig, ob die Geschwulst in der Nervenscheide entstanden oder erst später damit verwachsen ist, was oft schwer zu entscheiden, wenn das Sarkom bereits zum Theil seine Grenzen durchbrochen hat, vor unserer Beobachtung. Ich erinnere mich drei solcher Fälle, bei denen sich der Nerv ablösen liess.

Am 19. Februar 1872 operirte ich eine sehr magere Hausfrau mit ausserordentlich ungünstig gestaltetem Kreuzbein an einem 6 Monate alten, mannskopfgrossen Cystosarkom, welches mit Schmerzen in

1) Geschwälste. I. 356. Eine secundäre Verwachsung von Lipomen habe ich nur mit der Haut gesehen, wenn sie äusseren Schädlichkeiten ausgesetzt waren, wie ich das besonders bei den "Gurtlipomen" in der Taille der Fran oft gesehen habe. Eine derartige Verwachsung bestand hier nicht. 
der Nähe des linken Sitzknorren entstanden war und jetzt aufzubrechen drohte; leider ging die Geschwulst, mit der der Sitzknorren und der aufsteigende Ast des Sitzbeins resecirt werden musste, auch noch 2 Zoll hoch zwischen Mastdarm und Scheide hinauf. Der Nervus ischiadicus wurde 3 Zoll lang abpräparirt. Ohne alle Zufälle ging die grosse Wunde in eine schöne Granulationsfläche über; leider starb jedoch die schon vorher ausserordentlich magere Frau an Decubitus und schnell zunehmender Schwäche nach einigen Wochen.

Bei der Exstirpation eines zweiten mannskopfgrossen Sarkoms mehr abwärts am Oberschenkel - sie fand am 15. November 1874 bei einer durch viele Geburten sehr heruntergekommenen Dame aus dem Kanton Aargau in der Privatpraxis statt - worde der Hüftnerv, von dessen Scheide der Tumor auszugehen schien, 6 Zoll lang abgelöst; ebensoviel musste leider vorn von der starken Vena saphena magna excidirt werden, weil sie, wie anch ein Stiick vom Musc. sartorius, vom Tumor schon durchwachsen war. Diese Dame ging am 3. December an sebr acutem Trismus zu Grunde.

In einem dritten Fall (Herr Reg.- R. L. in Zürich) handelte es sich um ein grosses Sarkom der Ellenbenge, welches sich am Oberarm hinauf erstreckte. Der Medianus liess sich wie bei jenem Lipom vorn von ihm abtrennen. Allein leider musste schon die ganze infiltrirte vordere Ellenbogengelenkkapsel mit entfernt werden. Unter antiseptischer Behandlung kam die umfangreiche Wunde zwar ohne Zufälle zur Heilung, allein bald nach der Entlassung trat ein Recidiv ein, welches auch nach der Amputation sehr bald die Leiden des Kranken beendigt hat.

In allen diesen drei Fällen war - das zeigte sich erst so recht bei der Operation - das Sarkom leider nur zu weit über die Begrenzung in die Nachbarschaft vorgeschritten; in allen dreien liess sich jedoch der Nerv anscheinend in normalemVerhalten abtrennen; immerhin wurde ich ïber das ganz besonders heftige Auftreten von Tetanus und die so rapiden Rückfälle bei Herrn Reg.-R. L. stutzig.

Ich bin dann weiter gegangen und habe auch die eingebetteten Nervengeschwillste conservativ behandelt, ohne dass mir dabei ein fernerer Zufall vorgekommen wäre, obgleich der Nerv dabei oft noch mehr ins Gedränge kommt, wenn er sich nicht blos fächerförmig iiber eine Seite des meist spindelförmigen Tumors legt, sondern sich gleichmässig ïber denselben in allen Meridianen verbreitet. Es lässt sich dann oft nicht vermeiden, dass die Bündel einzeln nach der Operation und ringsum isolirt durch die Wundhöhle streichen.

Am ärgsten ging es in dieser Weise dem N. tib, post., aus dem 
ich 1878 ein ungefähr eigrosses Fibrom an der Grenze der Wade mit bestem Erfolg entfernte; der Kranke blieb geheilt, ohne dass von Tetanus dabei die Rede war.

Ueber einen nicht weniger schwierigen Fall haben schon die Herren Luning ${ }^{1}$ ) und Fischer in ibren Arbeiten berichtet. Am 13. Februar 1872 entfernte ich in der Privatpraxis ein kopfgrosses Sarkom aus dem Stamm des Obturatorius d., der auch in verschiedenen Meridianen mit seinen Fasern über die Geschwulst strich. Im Mai ging der Kranke gesund nach Haus. Allein schon im Juni bemerkte man ein Recidir bei dem Kranken, und so musste ich ihn am 4. December im Stadtspital des Kanton $\mathrm{Zug}$ der fürchterlicben Schmerzen wegen in der Hüfte exarticuliren; denn rings um den Knochen vom Knie bis zur Hüfte war der Schenkel spindelförmig entartet. Jedoch auch hier trat nach der Heilung bald ein Recidiv ein, dem der Kranke, ein Landwirth, am 17. September 1873 erlag. Vor der ersten Operation war die Krankheitsdauer 7 Monate.

Dann habe ich am 31. Juli 1869 ein eigrosses Sarkom, welches sich in zwei Monaten gebildet hatte, aus der Nervenscheide eines Astes des Plexus brachialis in der Acbsel mit grosser Mühe exstirpirt, wodurch sich die Motilität des Vorderarms besserte. Im $\mathrm{Fe}$ bruar 1872 trat ein Recidiv in der Achsel ein, welches leider seiner Ausdehnung wegen nicht mehr operabel war, als ich den Kranken wieder zu seben bekam. Er starb am 30. März 1873 an Erschöpfung infolge von Verjauchung und Anämie ohne Metastasen; die Section zeigte nichts als Lungenödem.

Endlich habe ich $1 \$ 79$ in der Klinik ein eingebettetes Sarkom aus dem Medianus am Oberarm exstirpirt; der Kranke wurde geheilt entlassen, ist aber auch kurz nach meinem Abgang 1881 einem Recidiv erlegen.

So habe ich in 8 Fällen (bei 4 eingebetteten Nervengeschwïlsten und bei 4 am Nerren anhaftenden), zum Theil unter Schwierigkeiten, jedoch jedesmal mit Erfolg, den Nerr mit seiner Scheide conserviren können; zwei ganz vorgeschrittene erlagen der Operation in den ersten Wochen, der eine an Erschöpfung, der andere an Tetanus.

1) August Lüning: Ueber die Blutung bei der Exarticulation des Oberschenkels und deren Vermeidung. Diss. inaug. Zürich bei Friedrich Schulthess. 1876. S. 45, und Siegfried Fischer, Ueber die Ursachen der Krebskrankheit und deren Heilbarkeit durch das Messer. Diss. inaug. Leipzig. Diese Zeitschrift. Bd. XIV. Fall 273. Man vergl. ferner hierin die Fälle 277 (Resectio ischii), 272 (Tetanusfall), und 265 (Neurosarkom des Plexus axillarisi. 
Bei gutartigen Fällen (2 yon den 8) lohnte sich das sehr, bei den bösartigen, den Sarkomen, habe ich mich dagegen nicht des Eindrucks erwehren können, als seien die Rückfälle besonders schnell eingetreten. Jedenfalls habe ich mit der n conservativen Schälmethode" keinen Fall mit Nervensarkom definitiv gebeilt, obgleich gerade in den 4 "eingebetteten "Fällen die gutartig verdickte Nervenscheide wie eine Kapsel den Tumor eingehüllt batte und nirgends bei sorgfältiger Untersuchung ergriffen zu sein schien. Bei den anhaftenden Sarkomen war freilich jedesmal schon die Kapsel bei der Operation nach anderer Richtung durchbrochen gefunden, so dass ich auf den definitiven Misserfolg schon eher gefasst sein musste. Scheint es nicht auch sonst, als ob die Operation der Sarkome um so sicherer definitiv lohnt, je fester sie eingekapselt, je weniger Nachbarorgane durchsetzt von Knötchen oder schon selbst hineingezogen vorgefunden werden? Am sichersten fährt der Kranke wohl stets, wenn man dann auch noch die gesunde Kapsel mit entfernen kann, und fallen, meine ich, dagegen leichte Mitverletzungen, bleibende Nachtheile wirklich gar nicht ins Gewicht!

Diese Erfahrungen haben mich von der Schälmethode bei Sarkomen in den Nerven zurückkommen lassen und habe ich deshalb in diesem Fall von Nerventumor am Halse mit Ueberzeugung und aller Absicht die ganzen Nervenstämme durchschnitten, soweit sie betheiligt waren. Denn schon aus der Grösse des Tumors allein machte ich nach dem, was ich bisher gesehen, den Schluss auf seine sarkomatöse Natur und irrte darin auch nicht.

Die Geschwulst wurde in Müller'scher Flüssigkeit gebärtet und im Mai von Herrn Assistenten Dr. Flohrsch iitz untersucht. In der Länge war sie dann anf $7 \mathrm{Cm}$., in der Dicke auf $4 \mathrm{Cm}$. geschrumpft. Hie und da hatten sich dadurch kleine erbsengrosse Erbabenheiten erhoben. In einer derben bindegewebigen Kapsel, aus der sich die Geschwulst leicht herausschälen liess, breiten sich die grossen Nervenstämme fächerförmig aus und verlaufen in dieser Weise um die Geschwulst herum, welche keine nervösen Theile einschliesst, sondern nach der Untersuchung des Herrn Dr. Flohrschütz ans einem grosszelligen Spindelsarkom besteht. Das sind also die Erfahrungen, die mich überhaupt von dem Ausschälen der Sarkome aus ihrer Kapsel, besonders aber an den Nerven zurtickgebracht haben.

Dass es nun freilich auch Fälle geben wird, in denen es selbst nicht mehr definitiv helfen wird, wenn man die Nervenfüsse des Sarkoms fingerbreit wie hier im Gesunden durchtrennt, das zeigen die 
zersplitternden Neurome vor Allem, das hat mich gleich mein erster Fall derart belehrt.

Am 25. September 1871 babe ich einem anscheinend gesunden Landwirth von 27 Jahren, Seb. R. aus Wetzikon im Kanton Zürich, den ganzen $\mathrm{N}$. ischiadicus links mit dem Anfang seiner beiden Aeste vom Schenkelhals bis zur Wade wegen eines mannskopfgrossen Tumors entfernt, der seit einem Jahr entstanden war, ihn Abends jetzt fiebern machte und vom Mai ab am Herumgehen verhinderte, ohne Nervenerscheinungen zu bewirken. Nach der Untersuchung von Prof. Eberth war es ein Fibroma myxosarcomatosum, bei dem die Enden des Tumors aus stark markhaltigen Nervenfasern bestanden. Der Nerv ging in den Tumor auf und liess sich nicht isoliren. Im Herbst konnte der Kranke wider Erwarten bereits mit einem Stock gehen; nur schlug dabei die linke Fusssohle etwas kräftiger auf als die rechte. Von Epilepsie 1) zeigte sich keine Spur und stellte ich ihn desbalb im Verein juingerer Aerzte in Zürich ") den Collegen B iermer, Gudden und Anderen vor. Leider zeigte sich am 11. Februar ein Recidiv an den Wundrändern; der Kranke starb ziemlich plötzlich am 24. Februar anscheinend an einer hypostatischeu Pneumonie; die Section ergab einen weissen Knoten in der Adventitia der Aorta, sowie Metastasen in den Lungen als Grund des terminalen Lungenödems. Dabei waren die Schnittenden des Ischiadicus, Peroneus und Tibialis aufgetrieben; endlich fanden sich aber zahlreiche Tumoren im Nervensystem, besonders im anderen N. ischiadicus und in der Cauda equina.

Es handelte sich also wohl schon vor der Operation um einen jener Fälle allgemeiner Neuromatose mit secundären Lungenmetastasen, die von vornherein jede Operation fruchtlos erscheinen lassen. Hier war mir bei Lebzeiten die grosse Zahl Warzen und Male, welche sich an seiner Haut vorfanden, aufgefallen; sonst hatte sich bei Lebzeiten kein Knoten ausser diesem spindelförmigen Tumor am Oberschenkel entdecken lassen.

Anders war es in einem zweiten Fall von allgemeiner Fibrosarcomatose des Nervensystems, bei dem von den 22 Tumoren, die sich ausschliesslich an den Nerven vorfanden, viele bei Diuck empfindliche sich schon äusserlich bemerken liessen. Da sie keine

1) Nach Brown-Séquard bewirkt Durchschneidung des Hüftnerven beim Meerschweinchen nach einiger Zeit Epilepsie. Vergl. Westphal, Berliner klin. Wochenschr. 15il. Nr. 35: ,Ueber künstliche Erzeugung der Epilepsie beim Meerschweinchen." S. 2.

2) Vergl. Schweizer Correspondenzblatt is71. 
nervösen Störungen machten und seit der Jugend bei dem beschränkten 49 Jabre alten Landarbeiter schon bestanden, so trat ibre Operation gar nicht in Frage. Der Kranke lag auf der inneren Abtheilung des Herrn Collegen Biermer und wurde ich nur wegen der bestehenden Strumastenose consultirt; allein ehe noch etwas geschehen, starb der Kranke in derselben Nacht plötzlich an Glottisödem infolge latenter, in die Speiseröhre perforirter Ringknorpelnekrose, wie Herr Dr. Seitz') in seinem interessanten Bericht über diesen Fall mitgetheilt hat.

Meinen dritten Fall von allgemeiner Neuromatose babe ich schon in meiner Arbeit ${ }^{2}$ ) "Ueber die Exstirpation gutartiger Bauchgewächse (die Laparektomie) "beschrieben. Da handelte es sich um einen hereditär belasteten jungen Italiener, welcher von Geburt mit Mälern und kleinen Geschwïlsten bedeckt war, die zum Theil jetzt sehr empfindlich geworden waren. Da die Exstirpation dreier Geschwülste Herrn Prof. Eberth bei der histologischen Untersuchung als Resultat ergaben, dass es reine Fibrome waren, hielt ich es zuletzt für meine Pflicht, mich auf Andringen der Familie auch an das colossale retroperitoneale Neuroma crurale zu machen, welches unter abendlichem Fieber und durch die steten Schmerzen den Kranken sehr heruntergebracht hatte. Es war ein kürbissgrosses Myxosarkom und so verjaucht, dass die Schälmethode insofern dabei scheiterte, als die Kapsel stïckweise ausgeräumt werden musste. Leider uberstand der Kranke den Eingriff nur kurze Zeit; bei der Section fanden sich noch einige kleine sarkomatöse Lymphdrisen neben der Wirbelsäule, sonst jedoch keine Metastasen vor. Todesursache war wohl jene secundäre Atrophie des $Z$ werchfells, über die ich dort berichtet habe.

Dies sind meine Erfahrungen über die Operation der sogenannten falschen Neurome; von den wabren babe ich mich seitdem mehrfacb mit den Stumpf- und Amputationsneuromen beschäftigt, eine dankbarere Aufgabe, wo aber die Schwierigkeit für den Operateur auf einem ganz anderen Gebiet liegt. Auf die Erbaltung der Nervenfunction kommt hier nichts an, im Gegentheil ibre Zerstörung ist unsere Aufgabe, wenn sich der Nervenstumpt durch Empfindlichkeit geltend gemacht, die scblimmsten Falls die heftigsten Formen von Neuralgie so und bei leisester Berïhrung darstellen kann, ja sogar zur Reflexepilepsie und ascendirend zu Hirnberden zu fübren scheint. Die Erhaltung des Nerven ist bier in der Regel ganz werthlos, ja

1) Vergl. Virchow's Archiv. 1871. Hd. 52. S.114.

2) Diese Zeitschrift. Bd. XIX. S. 32. Leipzig 1564. 
es fragt sich, ob eine weit hinaufgehende Exstirpation des Nervenstammes mit sammt dem Neurom den Erfolg nicht noch sicherer gestaltet.

Schwer ist oft nur die Frage zu entscheiden, wie man den Fall operativ angreifen soll, da durch die Amputationsnarben die örtlichen Verbältnisse so verändert sind, und man oft gar keine Sicherbeit hat, in welchem Nerv das lästige Neurom wohl stecken mag. Gerade die empfindlichsten lassen sich, wenn in der Narbe Knochen und Sehnen verwachsen sind, der tiefen Lage wegen kaum oder gar nicht durchfiblen.

Das schwerste Leiden derart zeigte mir ein junger oldenburger Kaufmann, Herr F. E., der in der Kindheit an Caries gelitten hatte und vor einigen Jahren am Oberschenkel von Wilms amputirt war. Die entsetzlichste Neuralgie im Stumpf zwang ihn, sein küustliches Bein wegzulassen; nur der reichliche Genuss von Bier betäubte etwas seine Schmerzen, die ihn ganz leistungsunfähig machten. Infolge des Bieres und der Unthätigkeit war er so stark geworden, dass man an dem prallen Stumpf selbst in der Narkose nichts durchfühlen konnte, zumal er mehrfach mit Narben besetzt war, Folge von fruchtlosen Operationen, die zum Theil noch Wilms, zum Theil andere Operateure der Neuralgie wegen vorgenommen batten.

Die Angabe, dass die Schmerzen oft scheinbar in den grossen Zeh ausstrahiten, veranlassten mich, den N. ischiadicus am Schenkelhals aufzusuchen, wo er jedenfalls noch am leichtesten zu finden sein musste. Von dort aus wurde er bis zur Narbe der Amputation blossgelegt und verfolgt, das heisst, da die Absetzung uber dem Knie stattgefunden hat, wurde der ganze mächtige Oberschenkel auf seiner Rückseite aufgeschnitten. Hinter der Narbe, dicht am Knochen fand sich ein Neurom in der Tiefe der Weichtheile, von der Grösse eines kleinen Apfels, welches mit sammt dem Hifftnerv bis zum Schenkelhals entfernt wurde. Die recht tiefe Wunde wurde mit allen antiseptischen Cautelen genäht, heilte aber bei der trotz aller Vorsicht doch eintretenden nächtlichen grossen Uniube des Kranken nicht ganz durch Verklebung. Wegen seines künstlichen Beins hat er mich noch nach Jahr und 'Tag einmal wieder aufgesucht.

Bei Syme'schen Stümpfen habe ich mehrmals, geleitet allein durch die bestätigende locale Druckempfindlichkeit, Neurome aufgesucht, die so nicht durehzufiihlen waren. Auf ihre Grösse müssen dabei die Erwartungen nicht zu sehr gespannt sein. An Leichen im Operationscurs babe ich bei alten Unterschenkelamputationen öfters 
bei der Präparation zwischen den Sehnen des Stumpfes mehrfache spindelförmige Neurome gefunden, etwa von der Dicke der nebenliegenden Sehnen, die auch manchmal durch Seitenäste unter sich und mit den Narben zusammenhingen. Ich habe mich in Erinnerung daran selten mit der Entfernung eines Neuroms bei Lebzeiten in solchen Fällen begnügt, sondern nach ähnlichen Seitenästen und Nachbarneuromen gesucht; meist mit Erfolg.

Dies sind meine bisherigen Erfahrungen über die Operation der Neurome und haben sie mich eben veranlasst, bei bösartigen Fällen meine conservative Schälmethode ganz wieder aufzugeben. Ich will hoffen, dass unser Kranker für die Erb'sche Lähmung, die er davon getragen, durch einen um so dauerhafteren Erfolg entschädigt wird.

Berlin, Juni $18 \$ 6$. 